\title{
RELATING GAMES OF MENGER, COUNTABLE FAN TIGHTNESS, AND SELECTIVE SEPARABILITY
}

\author{
STEVEN CLONTZ
}

\begin{abstract}
By adapting techniques of Arhangel'skii, Barman, and Dow, we may equate the existence of perfect-information, Markov, and tactical strategies between two interesting selection games. These results shed some light on Gruenhage's question asking whether all strategically selectively separable spaces are Markov selectively separable.
\end{abstract}

\section{INTRODUCTION}

Definition 1. The selection principle $S_{\text {fin }}(\mathcal{A}, \mathcal{B})$ states that given $A_{n} \in \mathcal{A}$ for $n<\omega$, there exist $B_{n} \in\left[A_{n}\right]^{<\omega}$ such that $\bigcup_{n<\omega} B_{n} \in \mathcal{B}$.

Definition 2. The selection game $G_{f i n}(\mathcal{A}, \mathcal{B})$ is the analogous game to $S_{f i n}(\mathcal{A}, \mathcal{B})$, where during each round $n<\omega$, Player I first chooses $A_{n} \in \mathcal{A}$, and then Player II chooses $B_{n} \in\left[A_{n}\right]^{<\omega}$. Player II wins in the case that $\bigcup_{n<\omega} B_{n} \in \mathcal{B}$, and Player I wins otherwise.

This game and property were first formally investigated by Scheepers in "Combinatorics of open covers" 9, which inspired a series of ten sequels with several co-authors. We may quickly observe that if II has a winning strategy for the game $G_{\text {fin }}(\mathcal{A}, \mathcal{B})$, then $S_{\text {fin }}(\mathcal{A}, \mathcal{B})$ will hold, but the converse need not follow.

The power of this selection principle and game comes from their ability to characterize several properties and games from the literature. Of interest to us are the following.

Definition 3. Let $\mathcal{O}_{X}$ be the collection of open covers for a topological space $X$. Then $S_{\text {fin }}\left(\mathcal{O}_{X}, \mathcal{O}_{X}\right)$ is the well-known Menger property for $X$ ( $M$ for short), and $G_{f i n}\left(\mathcal{O}_{X}, \mathcal{O}_{X}\right)$ is the well-known Menger game.

Definition 4. An $\omega$-cover $\mathcal{U}$ for a topological space $X$ is an open cover such that for every $F \in[X]^{<\omega}$, there exists some $U \in \mathcal{U}$ such that $F \subseteq U$.

Definition 5. Let $\Omega_{X}$ be the collection of $\omega$-covers for a topological space $X$. Then $S_{\text {fin }}\left(\Omega_{X}, \Omega_{X}\right)$ is the $\Omega$-Menger property for $X$ ( $\Omega M$ for short), and $G_{f i n}\left(\Omega_{X}, \Omega_{X}\right)$ is the $\Omega$-Menger game.

In [5. Theorem 3.9] it was shown that $X$ is $\Omega$-Menger if and only if $X^{n}$ is Menger for all $n<\omega$.

Definition 6. Let $\mathcal{B}_{X, x}$ be the collection of subsets $A \subset X$ where $x \in \operatorname{cl} A$. (Call $A$ a blade of $x$.) Then $S_{f i n}\left(\mathcal{B}_{X, x}, \mathcal{B}_{X, x}\right)$ is the countable fan tightness property for $X$ at $x$ (CFT $T_{x}$ for short), and $G_{f i n}\left(\mathcal{B}_{X, x}, \mathcal{B}_{X, x}\right)$ is the countable fan tightness game for $X$ at $x$. 
Definition 7. A space $X$ has countable fan tightness (CFT for short) if it has countable fan tightness at each point $x \in X$.

Definition 8. Let $\mathcal{D}_{X}$ be the collection of dense subsets of a topological space $X$. (So, $\mathcal{D}_{X} \subseteq \mathcal{B}_{X, x}$ for all $x \in X$.) Then $S_{\text {fin }}\left(\mathcal{D}_{X}, \mathcal{B}_{X, x}\right)$ is the countable dense fan tightness property for $X$ at $x\left(C D F T_{x}\right.$ for short), and $G_{f i n}\left(\mathcal{D}_{X}, \mathcal{B}_{X, x}\right)$ is the countable dense fan tightness game for $X$ at $x$.

Definition 9. A space $X$ has countable dense fan tightness (CDFT for short) if it has countable dense fan tightness at each point $x \in X$.

Note that $C F T \Rightarrow C D F T$ for any space $X$ as $\mathcal{D}_{X} \subseteq \mathcal{B}_{X, x}$.

The notion of countable fan tightness was first studied by by Arhangel'skii in [1]. A result of that paper showed that for $T_{3 \frac{1}{2}}$ spaces $X$, the countable fan tightness of the space of real-vaued continuous functions with pointwise convergence $C_{p}(X)$ is characterized by the $\Omega$-Menger property of $X$.

Definition 10. $S_{\text {fin }}\left(\mathcal{D}_{X}, \mathcal{D}_{X}\right)$ is the selective separability property for $X$ ( $S S$ for short), and $G_{f i n}\left(\mathcal{D}_{X}, \mathcal{D}_{X}\right)$ is the selective separability game for $X$.

Of course, one may easily observe that a selective separable space is separable. In 2] Barman and Dow demonstrated that all separable Frechet spaces are selectively separable. They were also able to produce a space which is selectively separable, but does not allow II a winning strategy in the selective separability game.

The object of this paper is to investigate the game-theoretic properties characterized by the presence of winning limited information strategies in these selection games.

Definition 11. A strategy for II in the game $G_{f i n}(\mathcal{A}, \mathcal{B})$ is a function $\sigma$ satisfying $\sigma\left(\left\langle A_{0}, \ldots, A_{n}\right\rangle\right) \in\left[A_{n}\right]^{<\omega}$ for $\left\langle A_{0} \ldots, A_{n}\right\rangle \in \mathcal{A}^{n+1}$. We say this strategy is winning if whenever I plays $A_{n} \in \mathcal{A}$ during each round $n<\omega$, II wins the game by playing $\sigma\left(\left\langle A_{0}, \ldots, A_{n}\right\rangle\right)$ during each round $n<\omega$. If a winning strategy exists, then we write II $\uparrow G_{\text {fin }}(\mathcal{A}, \mathcal{B})$.

Definition 12. A Markov strategy for II in the game $G_{f i n}(\mathcal{A}, \mathcal{B})$ is a function $\sigma$ satisfying $\sigma(A, n) \in\left[A_{n}\right]^{<\omega}$ for $A \in \mathcal{A}$ and $n<\omega$. We say this Markov strategy is winning if whenever I plays $A_{n} \in \mathcal{A}$ during each round $n<\omega$, II wins the game by playing $\sigma\left(A_{n}, n\right)$ during each round $n<\omega$. If a winning Markov strategy exists, then we write II $\underset{\text { mark }}{\uparrow} G_{f i n}(\mathcal{A}, \mathcal{B})$.

Notation 13. If $S_{\text {fin }}(\mathcal{A}, \mathcal{B})$ characterizes the property $P$, then we say II $\uparrow G_{\text {fin }}(\mathcal{A}, \mathcal{B})$ characterizes $P^{+}$("strategically $P^{\text {") }}$, and II $\underset{\text { mark }}{\uparrow} G_{f i n}(\mathcal{A}, \mathcal{B})$ characterizes $P^{+ \text {mark }}$ ("Markov $P$ "). Of course, $P^{+m a r k} \Rightarrow P^{+} \Rightarrow P$.

In this notation, Barman and Dow showed that $S S$ does not imply $S S^{+}$. Our goal is to make progress on the following question attributed to Gary Gruenhage:

Question 14. Does $S S^{+}$imply $S S^{+m a r k}$ ?

The solution is already known to be "yes" in the context of countable spaces 2]. However in general, winning strategies in selection games cannot be improved to be winning Markov strategies. In [4] the author showed that while $M^{+}$implies $M^{+m a r k}$ for second-countable spaces, there exists a simple example of a regular non-second-countable space which is $M^{+}$but not $M^{+ \text {mark }}$. 
RELATING GAMES OF MENGER, COUNTABLE FAN TIGHTNESS, AND SELECTIVE SEPARABILITß

\section{CFT, CDFT AND $S S$}

We begin by generalizing the following result:

Theorem 15 (Lem 2.7 of [2]). The following are equivalent for any topological space $X$.

- $X$ is $S S$.

- $X$ is separable and CDFT.

- $X$ has a countable dense subset $D$ where $C D F T_{x}$ holds for all $x \in D$.

Theorem 16. The following are equivalent for any topological space $X$.

- $X$ is $S S$ (resp. $\left.S S^{+}, S S^{+m a r k}\right)$.

- $X$ is separable and CDFT (resp. CDFT ${ }^{+}, C D F T^{+m a r k}$ ).

- $X$ has a countable dense subset $D$ where $C D F T_{x}$ (resp. $C D F T_{x}^{+}, C D F T_{x}^{+m a r k}$ ) holds for all $x \in D$.

Proof. We need only show that the final condition implies the first. Let $D=\left\{d_{i}\right.$ : $i<\omega\}$.

Let $\sigma_{i}$ be a witness for $C D F T_{d_{i}}^{+}$for each $i<\omega$. We define the strategy $\tau$ for the $S S$ game by

$$
\tau\left(\left\langle D_{0}, \ldots, D_{n}\right\rangle\right)=\bigcup_{i \leq n} \sigma_{i}\left(\left\langle D_{i}, \ldots, D_{n}\right\rangle\right) .
$$

Let $\left\langle D_{0}, D_{1}, \ldots\right\rangle \in \mathcal{D}_{X}^{\omega}$. By $C D F T_{d_{i}}^{+}$, we have

$$
d_{i} \in \overline{\bigcup_{i \leq n<\omega} \sigma_{i}\left(\left\langle D_{i}, \ldots, D_{n}\right\rangle\right)} \subseteq \overline{\bigcup_{i \leq n<\omega} \tau\left(\left\langle D_{0}, \ldots, D_{n}\right\rangle\right)} \subseteq \overline{\bigcup_{n<\omega} \tau\left(\left\langle D_{0}, \ldots, D_{n}\right\rangle\right)}
$$

and as $D \subseteq \overline{\bigcup_{n<\omega} \tau\left(\left\langle D_{0}, \ldots, D_{n}\right\rangle\right)}$ it follows that

$$
X \subseteq \bar{D} \subseteq \overline{\overline{\bigcup_{n<\omega} \tau\left(\left\langle D_{0}, \ldots, D_{n}\right\rangle\right)}}=\overline{\bigcup_{n<\omega} \tau\left(\left\langle D_{0}, \ldots, D_{n}\right\rangle\right)} .
$$

Therefore $\tau$ witnesses $S S^{+}$.

Now let $\sigma_{i}$ be a witness for $C D F T_{d_{i}}^{+ \text {mark }}$ for each $i<\omega$. We define the Markov strategy $\tau$ for the $S S$ game by

$$
\tau(D, n)=\bigcup_{i \leq n} \sigma_{i}(D, n-i) .
$$

Let $\left\langle D_{0}, D_{1}, \ldots\right\rangle \in \mathcal{D}_{X}^{\omega}$. By $C D F T_{d_{i}}^{+m a r k}$, we have

$$
d_{i} \in \overline{\bigcup_{i \leq n<\omega} \sigma_{i}\left(D_{n}, n-i\right)} \subseteq \overline{\bigcup_{i \leq n<\omega} \tau\left(D_{n}, n\right)} \subseteq \overline{\bigcup_{n<\omega} \tau\left(D_{n}, n\right)}
$$

and as $D \subseteq \overline{\bigcup_{n<\omega} \tau\left(D_{n}, n\right)}$ it follows that

$$
X \subseteq \bar{D} \subseteq \overline{\overline{\bigcup_{n<\omega} \tau(D, n)}}=\overline{\bigcup_{n<\omega} \tau(D, n)} .
$$

Therefore $\tau$ witnesses $S S^{+m a r k}$.

So amongst separable spaces, we see that $S S$ (resp. $S S^{+}, S S^{+m a r k}$ ) and $C D F T$ (resp. $C D F T^{+}, C D F T^{+m a r k}$ ) are equivalent. We now further bridge the gap between $C D F T$ and $C F T$ in the context of function spaces. Consider the following result of Arhangel'skii. 
Theorem 17 (1]). The following are equivalent for any $T_{3 \frac{1}{2}}$ topological space $X$.

- $X$ is $\Omega M$.

- $C_{p}(X)$ is $C F T$.

This result may similarly be generalized in a game theoretic sense. In addition, this proof will demonstrate the equivalence of $C F T$ and $C D F T$ in $C_{p}(X)$. It is unknown to the author whether Arhangel'skii used a strategy similar to the following proof in [1, but Sakai employed a similar technique in [8] to relate the $\Omega$-Rothberger and countable strong fan tightness properties (and essentially, the countable strong dense fan tightness property). Due to the difficulty in obtaining an English translation of [1, we reprove Arhangel'skii's theorem above in our more general context below.

Proposition 18. Any witness for $\Omega M$ (resp. $\Omega M^{+}, \Omega M^{+m a r k}$ ) may be improved such that any final sequence of the chosen finite subcollections is an $\omega$-cover.

Proof. Consider any sequence $\left\langle\mathcal{U}_{0}, \mathcal{U}_{1}, \ldots\right\rangle$ of open covers. For $\Omega M$, choose the witness $\left\langle\mathcal{F}_{n}^{n}, \mathcal{F}_{n+1}^{n}, \ldots\right\rangle$ for the final sequence $\left\langle\mathcal{U}_{n}, \mathcal{U}_{n+1}, \ldots\right\rangle$ for each $n<\omega$, and let $\mathcal{F}_{n}=\bigcup_{i \leq n} \mathcal{F}_{n}^{i}$. For $\Omega M^{+}$, the winning strategy $\sigma$ may be improved to $\tau$ where $\tau\left(\left\langle\mathcal{U}_{0}, \ldots, \mathcal{U}_{n}\right\rangle\right)=\bigcup_{i \leq n} \sigma\left(\left\langle\mathcal{U}_{i}, \ldots, \mathcal{U}_{n}\right\rangle\right)$. For $\Omega M^{+m a r k}$, the winning Markov strategy $\sigma$ may be improved to $\tau$ where $\tau(\mathcal{U}, n)=\bigcup_{i \leq n} \sigma(\mathcal{U}, i)$.

Definition 19. Let $X$ be a $T_{3 \frac{1}{2}}$ topological space. For $\mathbf{x} \in C_{p}(X), F \in[X]^{<\omega}$, and $\epsilon>0$, let

$$
[\mathbf{x}, F, \epsilon]=\left\{\mathbf{y} \in C_{p}(x):|\mathbf{y}(t)-\mathbf{x}(t)|<\epsilon \text { for all } t \in F\right\}
$$

give a basic open neighborhood of $\mathbf{x}$.

Lemma 20. Let $X$ be a $T_{3 \frac{1}{2}}$ topological space. If $X$ is $\Omega M$ (resp. $\left.\Omega M^{+}, \Omega M^{+m a r k}\right)$. then $C_{p}(X)$ is $C F T_{\mathbf{0}}$ (resp. $C F T_{\mathbf{0}}^{+}, C F T_{\mathbf{0}}^{+ \text {mark }}$ ).

Proof. For each $B \in \mathcal{B}_{C_{p}(X), \text { o define }}$

$$
\mathcal{U}_{n}(B)=\left\{\mathbf{x}^{\leftarrow}\left[\left(-\frac{1}{2^{n}}, \frac{1}{2^{n}}\right)\right]: \mathbf{x} \in B\right\} .
$$

Consider the finite set $F \in[X]^{<\omega}$. Since $\mathbf{0} \in \bar{B}$, choose $\mathbf{x} \in B \cap[\mathbf{0}, F, 1]$. It follows that $F \subseteq x^{\leftarrow}[(-1,1)] \in \mathcal{U}_{n}(B)$, so $\mathcal{U}_{n}(B)$ is an $\omega$-cover of $X$.

Consider the sequence of blades $\left\langle B_{0}, B_{1}, \ldots\right\rangle \in \mathcal{B}_{C_{p}(X), \mathbf{0}}^{\omega}$, and the corresponding sequence of $\omega$-covers $\left\langle\mathcal{U}_{0}\left(B_{0}\right), \mathcal{U}_{1}\left(B_{1}\right), \ldots\right\rangle \in \Omega_{X}^{\omega}$.

Assuming $X$ is $\Omega M$, choose a witness $\left\langle\mathcal{F}_{0}, \mathcal{F}_{1}, \ldots\right\rangle$ such that $\mathcal{F}_{n} \in\left[\mathcal{U}_{n}\left(B_{n}\right)\right]^{<\omega}$ and

$$
\bigcup_{i \leq n<\omega} \mathcal{F}_{n}
$$

is an $\omega$-cover of $X$ for all $i<\omega$. Now let

$$
F_{n}=\left\{\mathrm{x} \in B_{n}: \mathbf{x}^{\leftarrow}\left[\left(-\frac{1}{2^{n}}, \frac{1}{2^{n}}\right)\right] \in \mathcal{F}_{n}\right\} .
$$

We claim that $\mathbf{0} \in \overline{\bigcup_{n<\omega} F_{n}}$. Let $G \in[X]^{<\omega}, \epsilon>0$. Choose $i<\omega$ such that $\frac{1}{2^{i}}<\epsilon$ and then choose $i \leq n<\omega, \mathbf{x}^{\leftarrow}\left[\left(-\frac{1}{2^{n}}, \frac{1}{2^{n}}\right)\right] \in \mathcal{F}_{n}$ such that $G \subseteq \mathbf{x}^{\leftarrow}\left[\left(-\frac{1}{2^{n}}, \frac{1}{2^{n}}\right)\right]$ and therefore $G \subseteq \mathbf{x}^{\leftarrow}[(-\epsilon, \epsilon)]$. It follows that $\mathbf{x} \in F_{n} \cap[\mathbf{0}, G, \epsilon]$, and therefore every basic open neighborhood of $\mathbf{0}$ intersects $\bigcup_{n<\omega} F_{n}$. 
Assuming $X$ is $\Omega M^{+}$, choose a witness $\sigma$ such that

$$
\bigcup_{i \leq n<\omega} \sigma\left(\left\langle\mathcal{U}_{0}\left(B_{0}\right), \ldots, \mathcal{U}_{n}\left(B_{n}\right)\right\rangle\right)
$$

is an $\omega$-cover of $X$ for all $i<\omega$. Now let

$$
\tau\left(\left\langle B_{0}, \ldots, B_{n}\right\rangle\right)=\left\{\mathbf{x} \in B_{n}: \mathbf{x}^{\leftarrow}\left[\left(-\frac{1}{2^{n}}, \frac{1}{2^{n}}\right)\right] \in \sigma\left(\left\langle\mathcal{U}_{0}\left(B_{0}\right), \ldots, \mathcal{U}_{n}\left(B_{n}\right)\right\rangle\right)\right\} .
$$

We claim that $\mathbf{0} \in \overline{\bigcup_{n<\omega} \tau\left(\left\langle B_{0}, \ldots, B_{n}\right\rangle\right)}$. Let $G \in[X]^{<\omega}, \epsilon>0$. Choose $i<\omega$ such that $\frac{1}{2^{i}}<\epsilon$ and then choose $i \leq n<\omega, \mathbf{x}^{\leftarrow}\left[\left(-\frac{1}{2^{n}}, \frac{1}{2^{n}}\right)\right] \in \sigma\left(\left\langle\mathcal{U}_{0}\left(B_{0}\right), \ldots, \mathcal{U}_{n}\left(B_{n}\right)\right\rangle\right)$ such that $G \subseteq \mathbf{x}^{\leftarrow}\left[\left(-\frac{1}{2^{n}}, \frac{1}{2^{n}}\right)\right]$ and therefore $G \subseteq \mathbf{x}^{\leftarrow}[(-\epsilon, \epsilon)]$. It follows that $\mathbf{x} \in \tau\left(\left\langle B_{0}, \ldots, B_{n}\right\rangle\right) \cap[\mathbf{0}, G, \epsilon]$, and therefore every basic open neighborhood of $\mathbf{0}$ intersects $\bigcup_{n<\omega} \tau\left(\left\langle B_{0}, \ldots, B_{n}\right\rangle\right)$.

Assuming $X$ is $\Omega M^{+ \text {mark }}$, choose a witness $\sigma$ such that

$$
\bigcup_{i \leq n<\omega} \sigma\left(\mathcal{U}_{n}\left(B_{n}\right), n\right)
$$

is an $\omega$-cover of $X$ for all $i<\omega$. Now let

$$
\tau\left(B_{n}, n\right)=\left\{\mathbf{x} \in B_{n}: \mathbf{x}^{\leftarrow}\left[\left(-\frac{1}{2^{n}}, \frac{1}{2^{n}}\right)\right] \in \sigma\left(\mathcal{U}_{n}\left(B_{n}\right), n\right)\right\} .
$$

We claim that $\mathbf{0} \in \overline{\bigcup_{n<\omega} \tau\left(B_{n}, n\right)}$. Let $G \in[X]^{<\omega}, \epsilon>0$. Choose $i<\omega$ such that $\frac{1}{2^{i}}<\epsilon$ and then choose $i \leq n<\omega, \mathbf{x}^{\leftarrow}\left[\left(-\frac{1}{2^{n}}, \frac{1}{2^{n}}\right)\right] \in \sigma\left(\mathcal{U}_{n}\left(B_{n}\right), n\right)$ such that $G \subseteq$ $\mathbf{x}^{\leftarrow}\left[\left(-\frac{1}{2^{n}}, \frac{1}{2^{n}}\right)\right]$ and therefore $G \subseteq \mathbf{x}^{\leftarrow}[(-\epsilon, \epsilon)]$. It follows that $\mathbf{x} \in \tau\left(B_{n}, n\right) \cap[\mathbf{0}, G, \epsilon]$, and therefore every basic open neighborhood of $\mathbf{0}$ intersects $\bigcup_{n<\omega} \tau\left(B_{n}, n\right)$.

Lemma 21. Let $X$ be a $T_{3 \frac{1}{2}}$ topological space. If $C_{p}(X)$ is $C D F T_{\mathbf{0}}$ (resp. $C D F T_{\mathbf{0}}^{+}$, $C D F T_{\mathbf{0}}^{+ \text {mark }}$ ), then $X$ is $\Omega M$ (resp. $\Omega M^{+}, \Omega M^{+ \text {mark }}$ ).

Proof. For each $\mathcal{U} \in \Omega_{X}$ define

$$
D(\mathcal{U})=\left\{\mathbf{y} \in C_{p}(X): \mathbf{y}\left[X \backslash U_{\mathbf{y}, \mathcal{U}}\right]=\{1\} \text { for some } U_{\mathbf{y}, \mathcal{U}} \in \mathcal{U}\right\} .
$$

Consider the point $\mathbf{x} \in C_{p}(X)$ and its basic open neighborhood $[\mathbf{x}, G, \epsilon]$. If $\mathcal{U}$ is an $\omega$-cover of $X, G \subseteq U$ for some $U_{\mathbf{y}, \mathcal{U}} \in \mathcal{U}$. Since $X$ is $T_{3 \frac{1}{2}}, X \backslash U_{\mathbf{y}, \mathcal{U}}$ is closed, and $G$ is finite and disjoint from $X \backslash U_{\mathbf{y}, \mathcal{U}}$, we may choose some function $\mathbf{y} \in C_{p}(X)$ where $\mathbf{y}\left[X \backslash U_{\mathbf{y}}, \mathcal{U}\right]=\{1\}$ and $\mathbf{x}(t)=\mathbf{y}(t)$ for each $t \in G$. It follows $\mathbf{y} \in[\mathbf{x}, G, \epsilon] \cap D$, so $D(\mathcal{U})$ is dense in $C_{p}(X)$.

Consider the sequence of $\omega$-covers $\left\langle\mathcal{U}_{0}, \mathcal{U}_{1}, \ldots\right\rangle \in \Omega_{X}^{\omega}$, and the corresponding sequence of dense subsets $\left\langle D\left(\mathcal{U}_{0}\right), D\left(\mathcal{U}_{1}\right), \ldots\right\rangle \in \mathcal{D}_{C_{p}(X)}^{\omega}$.

Assuming $C_{p}(X)$ is $C D F T_{\mathbf{0}}$, choose a witness $\left\langle F_{0}, F_{1}, \ldots\right\rangle$ such that

$$
\mathbf{0} \in \overline{\bigcup_{n<\omega} F_{n}} .
$$

Now let

$$
\mathcal{F}_{n}=\left\{U_{\mathbf{y}, \mathcal{U}_{n}}: \mathbf{y} \in F_{n}\right\} \in\left[\mathcal{U}_{n}\right]^{<\omega} .
$$

We claim that $\bigcup_{n<\omega} \mathcal{F}_{n}$ is an $\omega$-cover. Let $G \in[X]<\omega$. The neighborhood $\left[\mathbf{0}, G, \frac{1}{2}\right]$ contains some point $\mathbf{y} \in F_{n}$ for some $n<\omega$. It follows that $U_{\mathbf{y}, \mathcal{U}_{n}} \in \mathcal{U}_{n}$ and $\mathbf{y}\left[X \backslash U_{\mathbf{y}, \mathcal{U}_{n}}\right]=\{1\}$. It follows that $G \cap\left(X \backslash U_{\mathbf{y}, \mathcal{U}_{n}}\right)=\emptyset$, and therefore $G \subseteq U_{\mathbf{y}, \mathcal{U}_{n}} \in$ $\mathcal{F}_{n}$. 
Assuming $C_{p}(X)$ is $C D F T_{\mathbf{0}}^{+}$, choose a witness $\sigma$ such that

$$
\mathbf{0} \in \overline{\bigcup_{n<\omega} \sigma\left(\left\langle D\left(\mathcal{U}_{0}\right), \ldots, D\left(\mathcal{U}_{n}\right)\right\rangle\right)} .
$$

Now let

$$
\tau\left(\left\langle\mathcal{U}_{0}, \ldots, \mathcal{U}_{n}\right\rangle\right)=\left\{U_{\mathbf{y}, \mathcal{U}_{n}}: \mathbf{y} \in \sigma\left(\left\langle D\left(\mathcal{U}_{0}\right), \ldots, D\left(\mathcal{U}_{n}\right)\right\rangle\right)\right\} \in\left[\mathcal{U}_{n}\right]^{<\omega} .
$$

We claim that $\bigcup_{n<\omega} \tau\left(\left\langle\mathcal{U}_{0}, \ldots, \mathcal{U}_{n}\right\rangle\right)$ is an $\omega$-cover. Let $G \in[X]^{<\omega}$. The neighborhood $\left[\mathbf{0}, G, \frac{1}{2}\right]$ contains some point $\mathbf{y} \in \sigma\left(\left\langle D\left(\mathcal{U}_{0}\right), \ldots, D\left(\mathcal{U}_{n}\right)\right\rangle\right)$ for some $n<\omega$. It follows that $U_{\mathbf{y}, \mathcal{U}_{n}} \in \mathcal{U}_{n}$ and $\mathbf{y}\left[X \backslash U_{\mathbf{y}, \mathcal{U}_{n}}\right]=\{1\}$. As a result $G \cap\left(X \backslash U_{\mathbf{y}, \mathcal{U}_{n}}\right)=\emptyset$, and therefore $G \subseteq U_{\mathbf{y}, \mathcal{U}_{n}} \in \tau\left(\left\langle\mathcal{U}_{0}, \ldots, \mathcal{U}_{n}\right\rangle\right)$.

Assuming $C_{p}(X)$ is $C D F T_{\mathbf{0}}^{+m a r k}$, choose a witness $\sigma$ such that

$$
\mathbf{0} \in \overline{\bigcup_{n<\omega} \sigma\left(D\left(\mathcal{U}_{n}\right), n\right)} .
$$

Now let

$$
\tau\left(\mathcal{U}_{n}, n\right)=\left\{U_{\mathbf{y}, \mathcal{U}_{n}}: \mathbf{y} \in \sigma\left(D\left(\mathcal{U}_{n}\right), n\right)\right\} \in\left[\mathcal{U}_{n}\right]^{<\omega} S .
$$

We claim that $\bigcup_{n<\omega} \tau\left(\mathcal{U}_{n}, n\right)$ is an $\omega$-cover. Let $G \in[X]^{<\omega}$. The neighborhood $\left[\mathbf{0}, G, \frac{1}{2}\right]$ contains some point $\mathbf{y} \in \sigma\left(D\left(\mathcal{U}_{n}\right), n\right)$ for some $n<\omega$. It follows that $U_{\mathbf{y}, \mathcal{U}_{n}} \in \mathcal{U}_{n}$ and $\mathbf{y}\left[X \backslash U_{\mathbf{y}, \mathcal{U}_{n}}\right]=\{1\}$. As a result $G \cap\left(X \backslash U_{\mathbf{y}, \mathcal{U}_{n}}\right)=\emptyset$, and therefore $G \subseteq U_{\mathbf{y}, \mathcal{U}_{n}} \in \tau\left(\mathcal{U}_{n}, n\right)$.

Theorem 22. The following are equivalent for any $T_{3 \frac{1}{2}}$ topological space $X$.

- $X$ is $\Omega M$ (resp. $\Omega M^{+}, \Omega M^{+ \text {mark }}$ ).

- $C_{p}(X)$ is $C F T$ (resp. $C F T^{+}, C F T^{+m a r k}$ ).

- $C_{p}(X)$ is $C D F T$ (resp. $C D F T^{+}, C D F T^{+m a r k}$ ).

Proof. Since $\mathcal{D}_{X} \subseteq \mathcal{B}_{X, x}$, the second condition trivially implies the first. As $C_{p}(X)$ is homogeneous, the $C(D) F T$ properties follow from $C(D) F T_{\mathbf{0}}$. So the result follows from the previous lemmas.

\section{A SPACE Which IS $C F T^{+}$BUT NOT $C D F T^{+ \text {mark }}$}

Our goal is to now demonstrate a space which is $C F T^{+}$, but not even $C D F T^{+m a r k}$. To do so, we will find a space $X$ which is $\Omega M^{+}$but not $\Omega M^{+m a r k}$, yielding $C_{p}(X)$ as our example.

Proposition 23. A space $X$ is compact if and only if for each $\omega$-cover $\mathcal{U}$ of $X$ and $n<\omega$, there exists a finite subcollection $\mathcal{F} \in[\mathcal{U}]^{<\omega}$ such that for each $F \in[X] \leq n$ there exists some $U \in \mathcal{F}$ where $F \subseteq U$.

Proof. Let $X$ and therefore $X^{n}$ be compact. Let $\mathcal{F}_{n}$ be the finite subcover of the open cover $\mathcal{U}_{n}=\left\{U^{n}: U \in \mathcal{U}\right\}$. Then $\mathcal{F}=\left\{U: U^{n} \in \mathcal{F}_{n}\right\}$ witnesses our desired result.

Lemma 24. The following are equivalent for a regular topological space $X$ :

- $X$ is $\sigma$-compact

- $X$ is $\Omega M^{+m a r k}$

- $X$ is $M^{+m a r k}$ 
Proof. The equivalence of $\sigma$-compact and $M^{+m a r k}$ in regular spaces was shown in [4]. As $\Omega M^{+ \text {mark }}$ trivially implies $M^{+ \text {mark }}$, we need only demonstrate that if $X=\bigcup_{n<\omega} K_{n}$ for $K_{n}$ compact and increasing, then $X$ is $\Omega M^{+m a r k}$.

We define $\sigma(\mathcal{U}, n)$ for each $\omega$-cover $\mathcal{U}$ and $n<\omega$ to witness Proposition 23 for $\mathcal{U}, K_{n}$, and $n$. It follows that for every sequence of $\omega$-covers $\left\langle\mathcal{U}_{0}, \mathcal{U}_{1}, \ldots\right\rangle$ and $F \in[X]^{<\omega}, F \in\left[K_{n}\right]^{\leq n}$ for some $n \geq|F|$, and thus there exists some $U \in \sigma\left(\mathcal{U}_{n}, n\right)$ where $F \subseteq U$. Therefore $\bigcup_{n<\omega} \sigma(\mathcal{U}, n)$ is an $\omega$-cover of $X$.

The reader may note that with this lemma, we may view Theorem 22 as a generalization of [3, Proposition 2.6].

Definition 25. Let $X$ be a topological space such that all countable sets are closed. Then $L(X)=X \cup\{\infty\}$ is the one-point Lindelöf-ication of $X$, with a basis given by the usual open sets of $X$ and the co-countable sets containing $\infty$.

Theorem 26. Let $\omega_{1}$ have the discrete topology. Then $L\left(\omega_{1}\right)$ is $\Omega M^{+}$but not $\Omega M^{+ \text {mark }}$.

Proof. The proof that $X=L\left(\omega_{1}\right)$ is not $\sigma$-compact (and therefore not $\Omega M^{+m a r k}$ ) is simply the fact that its countably infinite subsets are closed and discrete. We define the strategy $\sigma$ for II in $G_{f i n}\left(\Omega_{X}, \Omega_{X}\right)$ as follows.

For $n<\omega$ let $\sigma\left(\left\langle\mathcal{U}_{0}, \ldots, \mathcal{U}_{n}\right\rangle\right)=\left\{U\left(\left\langle\mathcal{U}_{0}, \ldots, \mathcal{U}_{n}\right\rangle\right)\right\}$, where

$$
U\left(\left\langle\mathcal{U}_{0}, \ldots, \mathcal{U}_{n}\right\rangle\right)=L\left(\omega_{1}\right) \backslash\left\{\alpha_{n, m}: m<\omega\right\}
$$

is a co-countable set containing $\{\infty\} \cup\left\{\alpha_{i, j}: i, j<n\right\}$.

Consider the arbitrary sequence of moves $\left\langle\mathcal{U}_{0}, \mathcal{U}_{1}, \ldots\right\rangle$ by I. For $F \in\left[L\left(\omega_{1}\right)\right]^{<\omega}$, choose $n<\omega$ such that

$$
F \cap\left\{\alpha_{i, j}: i, j<\omega\right\}=F \cap\left\{\alpha_{i, j}: i, j<n\right\} \subseteq U\left(\left\langle\mathcal{U}_{0}, \ldots, \mathcal{U}_{n}\right\rangle\right)
$$

It follows that as

$$
F \backslash\left\{\alpha_{i, j}: i, j<\omega\right\} \subseteq F \backslash\left\{\alpha_{n, m}: m<\omega\right\} \subseteq U\left(\left\langle\mathcal{U}_{0}, \ldots, \mathcal{U}_{n}\right\rangle\right)
$$

$F$ is a subset of $U\left(\left\langle\mathcal{U}_{0}, \ldots, \mathcal{U}_{n}\right\rangle\right)$, making $\bigcup_{n<\omega} \sigma\left(\left\langle\mathcal{U}_{0}, \ldots, \mathcal{U}_{n}\right\rangle\right)$ an $\omega$-cover.

Corollary 27. $C_{p}\left(L\left(\omega_{1}\right)\right)$ is $C F T^{+}$but not $C D F T^{+m a r k}$.

If $C_{p}\left(L\left(\omega_{1}\right)\right)$ were separable, it would be a negative solution to Question 14 . However, it is not.

Lemma 28 ([].6. For a $T_{3 \frac{1}{2}}$ topological space $X, C_{p}(X)$ is separable if and only if $X$ has a coarser separable metrizable topology.

Corollary 29. $C_{p}\left(L\left(\omega_{1}\right)\right)$ is not separable.

Proof. Every metrizable topological space has points $G_{\delta}$. However, if every neighborhood of $\infty$ is co-countable, $\{\infty\}$ cannot be the intersection of countably many open sets.

An affirmative answer to either of these questions would answer Question 14 negatively.

Question 30. Does there exist a separable subspace of $C_{p}\left(L\left(\omega_{1}\right)\right)$ which is not $C D F T^{+m a r k}$ ?

Question 31. Does there exist a non- $\sigma$-compact $\Omega M^{+}$space with a coarser separable metrizable topology? 


\section{Equivalence of Certain strategic And Markov Seleciton properties}

Barman and Dow previously demonstrated that $S S^{+}$is equivalent to $S S^{+m a r k}$ amongst countable spaces. A similar result by the author showed that $M^{+}$is equivalent to $M^{+m a r k}$ in second-countable spaces. The following result generalizes both.

Lemma 32. Let $\mathcal{A}$ be a family of sets where $|\bigcup \mathcal{A}| \leq \omega$ and let $\mathcal{B}$ be a family of sets closed under supersets. Then II $\uparrow G_{f i n}(\mathcal{A}, \mathcal{B})$ if and only if II $\underset{\text { mark }}{\uparrow} G_{\text {fin }}(\mathcal{A}, \mathcal{B})$.

Proof. Let $\sigma$ witness II $\uparrow G_{\text {fin }}(\mathcal{A}, \mathcal{B})$.

For $t \in \omega^{<\omega}$, suppose $Z_{s} \in \mathcal{A}$ has been defined for all $s \leq t$. Note then that $\left\{\sigma\left(\left\langle Z_{t \uparrow 1}, Z_{t \mid 2}, \ldots, Z_{t}, A\right\rangle: A \in \mathcal{A}\right\} \subseteq[\cup \mathcal{A}]^{<\omega}\right.$ and therefore is countable. So choose $Z_{t}-\langle n\rangle \in \mathcal{A}$ for $n<\omega$ such that $\left\{\sigma\left(\left\langle Z_{t \uparrow 1}, Z_{t \mid 2}, \ldots, Z_{t}, A\right\rangle: A \in \mathcal{A}\right\}=\right.$ $\left\{\sigma\left(\left\langle Z_{t \uparrow 1}, Z_{t \uparrow 2}, \ldots, Z_{t}, Z_{t \frown\langle n\rangle}\right\rangle\right): n<\omega\right\}$.

Let $b: \omega \rightarrow \omega^{<\omega}$ be a bijection, and define $\tau(A, n)=\sigma\left(\left\langle Z_{b(n) \uparrow 1}, Z_{b(n) \uparrow 2}, \ldots, Z_{b(n)}, A\right\rangle\right)$. Consider $\left\langle A_{0}, A_{1}, \ldots\right\rangle \in \mathcal{A}^{\omega}$.

Define $f \in \omega^{\omega}$ as follows. Let $n<\omega$ and suppose that $f \uparrow n$ has been already been defined. Then choose $f(n)<\omega$ such that $\sigma\left(\left\langle Z_{f \uparrow 1}, Z_{f \uparrow 2}, \ldots, Z_{f \uparrow n}, A_{b^{\leftarrow}(f\lceil n)}\right\rangle\right)=$ $\sigma\left(\left\langle Z_{f \uparrow 1}, Z_{f \nmid 2}, \ldots, Z_{f \uparrow n}, Z_{f \uparrow(n+1)}\right\rangle\right)$.

Since $\left\langle Z_{f \nmid 1}, Z_{f \nmid 2}, \ldots\right\rangle \in \mathcal{A}^{\omega}$, it follows that $\bigcup_{n<\omega} \sigma\left(\left\langle Z_{f \uparrow 1}, Z_{f \nmid 2}, \ldots, Z_{f\lceil n}\right\rangle\right) \in \mathcal{B}$. The result then follows from

$$
\begin{aligned}
& \bigcup_{n<\omega} \tau\left(A_{n}, n\right)=\bigcup_{n<\omega} \sigma\left(\left\langle Z_{b(n) \uparrow 1}, Z_{b(n) \uparrow 2}, \ldots, Z_{b(n)}, A_{n}\right\rangle\right) \\
& \supseteq \bigcup_{n<\omega, b(n)<f} \sigma\left(\left\langle Z_{b(n) \uparrow 1}, Z_{b(n) \uparrow 2}, \ldots, Z_{b(n)}, A_{n}\right\rangle\right) \\
& =\bigcup_{n<\omega, b(n)<f} \sigma\left(\left\langle Z_{f \uparrow 1}, Z_{f \uparrow 2}, \ldots, Z_{f \uparrow|b(n)|}, A_{b^{\leftarrow}(f \uparrow|b(n)|)}\right\rangle\right) \\
& =\bigcup_{n<\omega, b(n)<f} \sigma\left(\left\langle Z_{f \uparrow 1}, Z_{f \uparrow 2}, \ldots, Z_{f \uparrow|b(n)|}, Z_{f \uparrow(|b(n)|+1)}\right\rangle\right) \\
& =\bigcup_{n<\omega} \sigma\left(\left\langle Z_{f \uparrow 1}, Z_{f \uparrow 2}, \ldots, Z_{f \uparrow n}\right\rangle\right) \\
& \in \mathcal{B}
\end{aligned}
$$

as $\mathcal{B}$ is closed under supersets.

Corollary 33 ([3]). A countable space is $S S^{+}$if and only if it is $S S^{+m a r k}$.

Proof. $\cup \mathcal{D}_{X}=X$ is countable, and any set containing a dense set is dense.

Corollary 34. A second-countable space is $\Omega M^{+}$if and only if it is $\Omega M^{+m a r k}$.

Proof. First note that any witness for $\Omega M^{+}$is a witness for II $\uparrow G_{f i n}\left(\Omega_{X}^{*}, \Omega_{X}^{*}\right)$. Fix a countable base $\mathcal{B}$ for $X$ closed under finite unions, and let $\Omega_{X}^{*}$ be the collection of $\omega$-covers of $X$ which only use basic open sets. Then $\bigcup \Omega_{X}^{*}=\mathcal{B}$ is countable, and any set containing an $\omega$-cover is an $\omega$-cover; therefore II $\uparrow G_{f i n}\left(\Omega_{X}^{*}, \Omega_{X}^{*}\right)$ if and only if II $\uparrow_{\text {mark }} G_{f i n}\left(\Omega_{X}^{*}, \Omega_{X}^{*}\right)$.

So let $\sigma$ witness II $\underset{\text { mark }}{\uparrow} G_{f i n}\left(\Omega_{X}^{*}, \Omega_{X}^{*}\right)$. For each $\omega$-cover $\mathcal{U}$ and finite set $F \in$ $[X]^{<\omega}$, choose $U_{\mathcal{U}, F} \in \mathcal{U}$ such that $F \subseteq U$. Then choose a basic open set $U_{\mathcal{U}, F}^{*}$ such 
that $F \subseteq U_{\mathcal{U}, F}^{*} \subseteq U_{\mathcal{U}, F}$. Finally, for each $\omega$-cover $\mathcal{U}$, let

$$
\mathcal{U}^{*}=\left\{U_{\mathcal{U}, F}^{*}: F \in[X]^{<\omega}\right\} \in \Omega_{X}^{*} .
$$

Define the strategy $\tau$ such that $\tau(\mathcal{U}, n)=\left\{U_{\mathcal{U}, F}: U_{\mathcal{U}, F}^{*} \in \sigma\left(\mathcal{U}^{*}, n\right)\right\}$. For any sequence of $\omega$-covers $\left\langle\mathcal{U}_{0}, \mathcal{U}_{1}, \ldots\right\rangle$, it follows that $\bigcup_{n<\omega} \sigma\left(\mathcal{U}_{n}^{*}, n\right)$ is an $\omega$-cover, and therefore $\bigcup_{n<\omega} \tau\left(\mathcal{U}_{n}, n\right)$ is an $\omega$-cover also.

Corollary 35 (4]). A second-countable space is $M^{+}$if and only if it is $M^{+m a r k}$.

Proof. An easy adapatation of the preceding proof, replacing $\omega$-covers with open covers, and replacing $F \in[X]^{<\omega}$ with $x \in X$.

\section{Strong VARiants of SELECTiOn PRINCPles AND games}

Let $S_{1}(\mathcal{A}, \mathcal{B}), G_{1}(\mathcal{A}, \mathcal{B})$ be the natural variants of $S_{\text {fin }}(\mathcal{A}, \mathcal{B}), G_{\text {fin }}(\mathcal{A}, \mathcal{B})$ where each choice by II must either be a single element or singleton (whichever is more convenient for the proof at hand), rather than a finite set. Convention calls for denoting these as strong versions of the corresponding selection princples and games, although the "strong Menger" property is commonly known as "Rothberger" who first investigated the principle in [7. We will thus call "strong $\Omega$-Menger" " $\Omega$ Rothberger" and shorten it with $\Omega R$, and otherwise attach the prefix "s" when abbreviating to all other strong variants.

Theorem 36. The following are equivalent for any topological space $X$.

- $X$ is $s S S$ (resp. $\left.s S S^{+}, s S S^{+m a r k}\right)$.

- $X$ is separable and $s C D F T$ (resp. $\left.s C D F T^{+}, s C D F T^{+m a r k}\right)$.

- X has a countable dense subset D where $s C D F T_{x}$ (resp. sCDFT $T_{x}^{+}, s C D F T_{x}^{+m a r k}$ ) holds for all $x \in D$.

Proof. We need only show that the final condition implies the first. Let $D=\left\{d_{i}\right.$ : $i<\omega\}$.

Let $\left\{D_{m, n} \in \mathcal{D}_{X}: m, n<\omega\right\}$, and let $x_{i, n} \in D_{i, n}$ witness $s C D F T_{d_{i}}$, so

$$
d_{i} \in \overline{\left\{x_{i, n}: n<\omega\right\}} \subseteq \overline{\left\{x_{m, n}: m, n<\omega\right\}}
$$

and as $D \subseteq \overline{\left\{x_{m, n}: m, n<\omega\right\}}$ it follows that

$$
X \subseteq \bar{D} \subseteq \overline{\overline{\left\{x_{m, n}: m, n<\omega\right\}}}=\overline{\left\{x_{m, n}: m, n<\omega\right\}} .
$$

Therefore $x_{m, n} \in D_{m, n}$ witnesses $s S S$.

Let $\sigma_{i}$ be a witness for $s C D F T_{d_{i}}^{+}$for each $i<\omega$. Define $p: \omega \rightarrow \omega$ partition $\omega$ into infinite sets $\{p(i): i<\omega\}$. For a nonempty finite sequence $t$, let $t^{\prime}$ be its subsequence removing all terms of index $n$ such that $p(n) \neq p(|t|-1)$. (Note that this process preserves the final term.) We then define the strategy $\tau$ by

$$
\tau(t)=\sigma_{p(|t|-1)}\left(t^{\prime}\right) .
$$

Let $\alpha \in \mathcal{D}_{X}^{\omega}$, and let $\alpha_{i}$ be its subsequence removing all terms of index $n$ such that $p(n) \neq i$. By $s C D F T_{d_{i}}^{+}$, we have

$d_{i} \in \overline{\left\{\sigma_{i}\left(\alpha_{i} \uparrow(n+1)\right): n<\omega\right\}}=\overline{\left\{\tau(\alpha \uparrow(n+1)): n \in p^{\leftarrow}(i)\right\}} \subseteq \overline{\{\tau(\alpha \uparrow(n+1)): n<\omega\}}$ and as $D \subseteq \overline{\{\tau(\alpha \uparrow(n+1)): n<\omega\}}$ it follows that

$$
X \subseteq \bar{D} \subseteq \overline{\overline{\{\tau(\alpha \uparrow(n+1)): n<\omega\}}}=\overline{\{\tau(\alpha \uparrow(n+1)): n<\omega\}} .
$$


Therefore $\tau$ witnesses $S S^{+}$.

Let $\sigma_{i}$ be a witness for $s C D F T_{d_{i}}^{+ \text {mark }}$ for each $i<\omega$. Define $p: \omega \rightarrow \omega$ partition $\omega$ into infinite sets $\{p(i): i<\omega\}$. We then define the Markov strategy $\tau$ by

$$
\tau(D, n)=\sigma_{p(n)}(D,|\{m \in p(n): m \leq n\}|) .
$$

Let $\alpha \in \mathcal{D}_{X}^{\omega}$, and let $\alpha_{i}$ be its subsequence removing all terms of index $n$ such that $p(n) \neq i$. By $s C D F T_{d_{i}}^{+ \text {mark }}$, we have

$$
d_{i} \in \overline{\left\{\sigma_{i}\left(\alpha_{i}(n), n\right): n<\omega\right\}}=\overline{\left\{\tau(\alpha(n), n): n \in p^{\leftarrow}(i)\right\}} \subseteq \overline{\{\tau(\alpha(n), n): n<\omega\}}
$$

and as $D \subseteq \overline{\{\tau(\alpha(n), n): n<\omega\}}$ it follows that

$$
X \subseteq \bar{D} \subseteq \overline{\overline{\{\tau(\alpha(n), n): n<\omega\}}}=\overline{\{\tau(\alpha(n), n): n<\omega\}} .
$$

Therefore $\tau$ witnesses $S S^{+ \text {mark }}$.

As mentioned earlier, the following is a result of Sakai:

Theorem $37([8])$. The following are equivalent for any $T_{3 \frac{1}{2}}$ topological space $X$.

- $X$ is $\Omega R$.

- $C_{p}(X)$ is $s C F T$.

- $C_{p}(X)$ is $s C D F T$.

The corresponding game-theoretic results also hold.

Theorem 38. The following are equivalent for any $T_{3 \frac{1}{2}}$ topological space $X$.

- $X$ is $\Omega R$ (resp. $\left.\Omega R^{+}, \Omega R^{+m a r k}\right)$.

- $C_{p}(X)$ is $s C F T$ (resp. $s C F T^{+}, s C F T^{+m a r k}$ ).

- $C_{p}(X)$ is sCDFT (resp. sCDFT ${ }^{+}, s C D F T^{+m a r k}$ ).

Proof. In Lemmas 20 and 21. $|\tau(t)|=|\sigma(t)|$. Therefore they may be extended to the strong cases requiring $|\tau(t)|=|\sigma(t)|=1$, which yields our result.

Theorem 39. Let $\omega_{1}$ have the discrete topology. Then $L\left(\omega_{1}\right)$ is $\Omega R^{+}$but not $\Omega M^{+m a r k}$.

Proof. The strategy constructed in Theorem 26 is a witness.

Corollary 40. $C_{p}\left(L\left(\omega_{1}\right)\right)$ is $s C F T^{+}$but not $C D F T^{+m a r k}$.

Lemma 41. Let $\mathcal{A}$ be a family of sets where $|\bigcup \mathcal{A}| \leq \omega$ and let $\mathcal{B}$ be a family of sets closed under supersets. Then II $\uparrow G_{1}(\mathcal{A}, \mathcal{B})$ if and only if $\operatorname{II} \underset{\text { mark }}{\uparrow} G_{1}(\mathcal{A}, \mathcal{B})$.

Proof. In the proof of Lemma 32, $|\tau(t)|=|\sigma(t)|$.

Corollary 42. A countable space is $s S S^{+}$if and only if it is $s S S^{+m a r k}$.

Corollary 43. A second-countable space is $\Omega R^{+}$if and only if it is $\Omega R^{+m a r k}$.

Corollary 44. A second-countable space is $R^{+}$if and only if it is $R^{+m a r k}$.

\section{ACKnowledGements}

The author would like to thank Ziqin Feng and Gary Gruenhage for their helpful comments on the topic of $C_{p}\left(L\left(\omega_{1}\right)\right)$. 
RELATING GAMES OF MENGER, COUNTABLE FAN TIGHTNESS, AND SELECTIVE SEPARABILITIY

\section{REFERENCES}

[1] A. V. Arkhangel'ski1. Hurewicz spaces, analytic sets and fan tightness of function spaces. Dokl. Akad. Nauk SSSR, 287(3):525-528, 1986.

[2] Doyel Barman and Alan Dow. Selective separability and $\mathrm{SS}^{+}$. Topology Proc., 37:181-204, 2011.

[3] Doyel Barman and Alan Dow. Proper forcing axiom and selective separability. Topology Appl., 159(3):806-813, 2012

[4] Steven Clontz. Applications of limited information strategies in Menger's game (preprint). 2015.

[5] Winfried Just, Arnold W. Miller, Marion Scheepers, and Paul J. Szeptycki. The combinatorics of open covers. II. Topology Appl., 73(3):241-266, 1996.

[6] Robert A. McCoy and Ibula Ntantu. Topological properties of spaces of continuous functions, volume 1315 of Lecture Notes in Mathematics. Springer-Verlag, Berlin, 1988.

[7] Fritz Rothberger. Eine verschrfung der eigenschaft c. Fundamenta Mathematicae, 30(1):50-55, 1938.

[8] Masami Sakai. Property $C^{\prime \prime}$ and function spaces. Proc. Amer. Math. Soc., 104(3):917-919, 1988.

[9] Marion Scheepers. Combinatorics of open covers. I. Ramsey theory. Topology Appl., 69(1):3162, 1996.

Department of Mathematics and Statistics, The University of South Alabama, MoBILE, AL 36606

E-mail address: steven.clontz@gmail.com 